\title{
Use of the cross-type dowels when connecting elements of timber bridges
}

\author{
Anatoly Popov ${ }^{1, *}$, and Vladimir Zinoviev ${ }^{1}$ \\ ${ }^{1}$ Siberian Transport University, 630049 Novosibirsk, Russia
}

\begin{abstract}
The use of cross-type dowels that are battered down with a gunshot method makes it possible to reduce labor intensity and speed up the process of erection and reconstruction of timber bridges. Objective of this paper is to develop a methodology for estimation of the permissible distance between dowels. For this purpose, the method of holographic interferometry in colliding beams was used. The resulting equations were obtained for the patterns of interference fringes observed in reflected light transmitted through the hologram. The investigations showed that the shear component of the strain tensor decreases exponentially with distance from the dowel. The concept of deformation attenuation constant was introduced, i.e. the distance at which the deformation decreases by e times. It is recommended to set dowels at a distance equal to eight attenuation constants.
\end{abstract}

\section{Introduction}

This paper [1] proposes to use dowels with $\mathrm{x}$-shaped cross-section that are hammered by means of mounting guns. The gunshot method of making doweled connections can be fully appreciated by the builders who construct timber bridges in hard-to-reach areas. The spread of this method is promoted by the widespread use of mounting guns in the construction for the attachment of steel parts to brick or concrete structures. When hammering crosssectioned dowels with knife sharpening with the mounting guns, a dense mortise is formed, which increases the reliability of the connection [2-3]. Until now, no methodology has been developed for determination of the permissible distances between dowels.

The study of the strain state of wood is complicated because of the large anisotropy of the material [4]. The optical methods for studying the strain state are used in the study of products made of various materials under various loading methods [5-6]. For the experimental determination of the permissible distance between the axes of the dowels, it is proposed to use the method of recording of holograms proposed by Yu. N. Denisyuk [7$11]$.

\footnotetext{
* Corresponding author: 47604@mail.ru
} 


\section{Study method}

To derive the resulting equations of the patterns of the bands reconstructed by a doubleexposure hologram, we consider the optical scheme for hologram recording shown in Figure 1. The path of the beams during the first exposure is represented by solid lines. During the second exposure, the lines are dotted. The photographic plate is located in front of the studied section of the product and is illuminated by a plane coherent light (Fig. 1). The pattern of the bands, which is recorded in the light passing through the recording medium, shall be determined by the interference of waves reflected from the surface of the product.

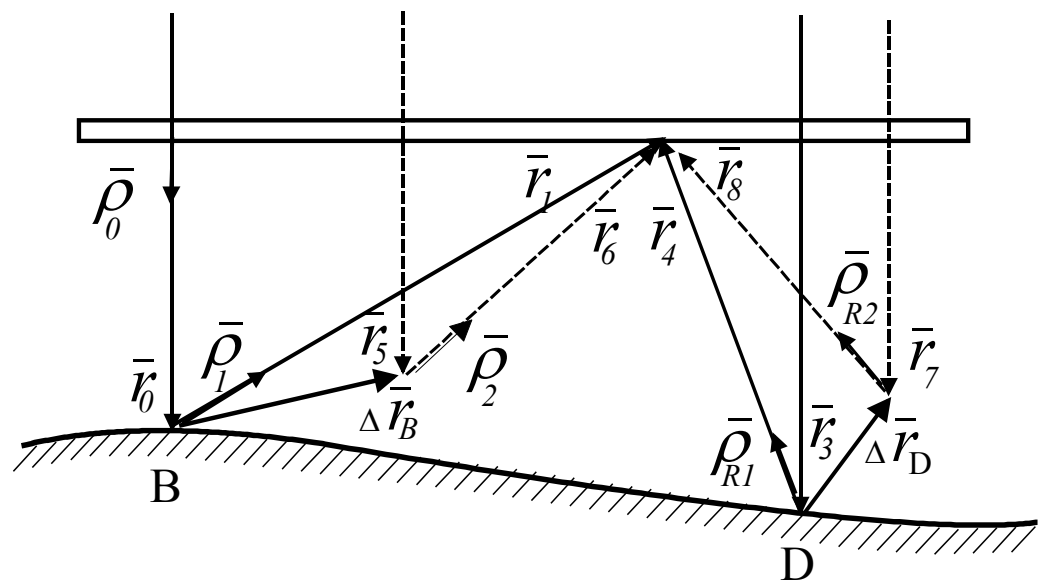

Fig. 1. Scheme of exposure optical path.

In the light reflected by a hologram, patterns are recorded that depend on the interference of the incident and reflected waves. Let us assume that $\vec{A}_{R 1}$ is the wave vector of the reference wave, and $\vec{A}_{1}$ is the same for the object one. A photographic plate is illuminated by a light wave $\vec{A}_{I}$ :

$$
\vec{A}_{I}=\vec{A}_{1}+\vec{A}_{R 1}
$$

The intensity of light is equal to the square of the modulus of the vector:

$$
I_{I}=\vec{A}_{I} \cdot \vec{A}_{I}^{*}=\vec{A}_{1}^{*} \cdot \vec{A}_{1}+\vec{A}_{R 1}^{*} \cdot \vec{A}_{1}+\vec{A}_{1}^{*} \cdot \vec{A}_{R 1}+\vec{A}_{R 1}^{*} \cdot \vec{A}_{R 1}
$$

Hereinafter, the symbol * denotes complex conjugation. Energy will enter the recording medium:

$$
E_{I}=I_{I} t_{1}
$$

Here $t_{1}$ is the time of exposure.

Similarly to the second exposure: 


$$
\vec{A}_{I I}=\vec{A}_{2}+\vec{A}_{R 2} ; I_{I I}=\vec{A}_{I I} \cdot \vec{A}_{I I}^{*} ; E_{I I}=I_{I I} t_{2}
$$

Here $\vec{A}_{2}$ and $\vec{A}_{R 2}$ are wave vectors of the object and reference wave; $A_{I I}$-light wave illuminating the photographic plate; $t_{2}$ - second exposure time.

The coefficient of amplitude transmission $T$ of the processed photographic plate is proportional to the obtained energy:

$$
T \approx E=I_{I} t_{1}+I_{\text {II }} t_{2}
$$

If the hologram is illuminated with light with a wave vector $\vec{A}_{R C}$, then it will reconstruct the light waves with the vector $\vec{A}_{B}$ :

$$
\begin{aligned}
& \vec{A}_{B}=T \vec{A}_{R C}=\left(\vec{A}_{I}^{*} \cdot \vec{A}_{I} t_{1}+\vec{A}_{I I}^{*} \cdot \vec{A}_{I I} t_{2}\right) \cdot \vec{A}_{R C}= \\
& {\left[\left(\vec{A}_{1}^{*} \cdot \vec{A}_{1} t_{1}+\vec{A}_{2}^{*} \cdot \vec{A}_{2} t_{2}+\vec{A}_{R 1}^{*} \cdot \vec{A}_{R 1} \cdot t_{1}+\vec{A}_{R 2}^{*} \cdot \vec{A}_{R 2} t_{2}\right)+\right.} \\
& +\left(\vec{A}_{R 1}^{*} \cdot \vec{A}_{1} t_{1}+\vec{A}_{R 2}^{*} \cdot \vec{A}_{2} t_{2}\right)+\left(\vec{A}_{1}^{*} \cdot \vec{A}_{R 1} t_{1}+\vec{A}_{2}^{*} \cdot \vec{A}_{R 2} t_{2}\right] \cdot \vec{A}_{R C}^{*}
\end{aligned}
$$

Further, we will consider only the terms enclosed in the second brackets, they describe the virtual image, the third brackets describe the real one, and the first brackets describe the non-diffracted light:

$$
\vec{A}_{B M}=\left[\vec{A}_{R 1}^{*} \cdot \vec{A}_{1} t_{1}+\vec{A}_{R 2}^{*} \cdot \vec{A}_{2} t_{2}\right] \cdot \vec{A}_{R C}
$$

The restored image will have an intensity:

$$
\begin{aligned}
& I=\left\{\left[\vec{A}_{R 1}^{*} \cdot \vec{A}_{1} t_{1}+\vec{A}_{R 2}^{*} \cdot \vec{A}_{2} t_{2}\right] \vec{A}_{R C}\right\} \cdot\left\{\left[\vec{A}_{R 1}^{*} \cdot \vec{A}_{1} t_{1}+\vec{A}_{R 2}^{*} \cdot \vec{A}_{2} t_{2}\right] \vec{A}_{R C}\right\}^{*}= \\
& =\left[\vec{A}_{R 1}^{*} \cdot \vec{A}_{1} t_{1}+\vec{A}_{R 2}^{*} \cdot \vec{A}_{2} t_{2}\right] \cdot\left[\vec{A}_{R 1}^{*} \cdot \vec{A}_{1} t_{1}+\vec{A}_{R 2}^{*} \cdot \vec{A}_{2} t_{2}\right]^{*} \cdot I_{R C} ;
\end{aligned}
$$

Since, $I_{R C}$ - the intensity of the reconstructing wave affects only the brightness of the image and having assumed $t_{1}=t_{2}=t$, then we will not take into account these parameters in the derivation of the resulting equations. In a complex form, the light wave vectors are represented as follows:

$$
\begin{gathered}
\vec{A}_{1}=A_{1} e^{i\left(-\vec{K}_{1} \cdot \vec{r}_{1}+\varphi_{1}\right)} ; \vec{A}_{R 1}=\vec{A}_{R 1} e^{i\left(-\vec{K}_{R 1} \cdot \vec{r}_{1}+\varphi_{R 1}\right)} \\
\vec{A}_{2}=A_{2} e^{i\left(-\vec{K}_{2} \cdot \vec{r}_{2}+\varphi_{2}\right)} ; \vec{A}_{R 2}=\vec{A}_{R 2} e^{i\left(-\vec{K}_{R 2} \cdot \vec{r}_{2}+\varphi_{R 2}\right)}
\end{gathered}
$$

Here, the indices of the amplitudes $A_{k}(k=1, \mathrm{R} 1,2, \mathrm{R} 2)$, wave vectors $\vec{K}_{k}$ and initial phases $\varphi_{k}$ indicate to which light vector they relate, $\vec{r}_{i}$ - the corresponding radius vectors. Let us denote: 


$$
\begin{aligned}
& -\vec{K}_{1} \cdot \vec{r}_{1}+\vec{K}_{R 1} \cdot \vec{r}_{1}+\varphi_{1}-\varphi_{R 1}=\alpha \\
& -\vec{K}_{2} \cdot \vec{r}_{2}+\vec{K}_{R 2} \cdot \vec{r}_{2}+\varphi_{2}-\varphi_{R 2}=\beta
\end{aligned}
$$

Then, if we assume that $A_{1} A_{R 1} \approx A_{2} A_{R 2}=A^{2}$ :

$$
\vec{A}_{R 1} \cdot \vec{A}_{1}=A^{2} e^{i \alpha} ; \vec{A}_{R 2} \cdot \vec{A}_{2}=A^{2} e^{i \beta}
$$

In the virtual image, the intensity of light is described by the expression:

$$
I \approx\left(e^{i \alpha}+e^{i \beta}\right)^{*} \cdot\left(e^{i \alpha}+e^{i \beta}\right)=2+2 \cos (\alpha-\beta)
$$

Maximum occurs under the following condition:

$$
\alpha-\beta=2 \pi N
$$

Having substituted formula (10) into equation (13) and multiplying it by $\frac{\lambda}{2 \pi}$, we obtain the following equation:

$$
-\vec{\rho}_{1} \cdot \vec{r}_{1}+\vec{\rho}_{R 1} \cdot \vec{r}_{1}+\left(\varphi_{1}-\varphi_{R 1}\right) \frac{\lambda}{2 \pi}+\vec{\rho}_{2} \cdot \vec{r}_{2}-\vec{\rho}_{R 2} \cdot \vec{r}_{2}-\left(\varphi_{2}-\varphi_{R 2}\right) \frac{\lambda}{2 \pi}=N \lambda
$$

Here $N$ is the number of the interference fringe; $\lambda$ - length of the light wave; $\vec{\rho}_{k}$ - unit vectors directed along light waves.

The phase $\varphi_{1}-\varphi_{R 1}$ and $\varphi_{2}-\varphi_{R 2}$ differences in equation (14) are also constant and do not affect the distance between the bands. Therefore, they will not be taken into account. Then equation (14) takes the form:

$$
\left(-\vec{\rho}_{1} \cdot \vec{r}_{1}+\vec{\rho}_{R 1} \cdot \vec{r}_{1}\right)-\left(-\vec{\rho}_{2} \cdot \vec{r}_{2}+\vec{\rho}_{R 2} \cdot \vec{r}_{2}\right)=N \lambda
$$

Let us denote $\Delta_{1}$, the terms in the first brackets (the path difference between the reference and object beam obtained at the first exposure), and in the second brackets $\Delta_{2}$ (the difference in the path of the same beams in the second exposure). Then, equation (15) takes the following form:

$$
\Delta_{1}-\Delta_{2}=N \lambda
$$

In accordance with Figure 1, the recording medium is illuminated by a plane wave with a unit vector $\vec{\rho}_{0}$, and the path difference of the light beams is calculated from it. The light waves corresponding to the first exposure are represented by solid lines, the second exposure - by dotted lines. It can be seen from the optical scheme that

$$
\begin{gathered}
\vec{r}_{5}=\vec{r}_{0}+\vec{W}_{B} ; \quad \vec{r}_{6}=\vec{r}_{1}-\Delta \vec{r}_{B} ; \vec{r}_{7}=\vec{r}_{3}+\vec{W}_{D} \\
\vec{r}_{8}=\vec{r}_{4}-\Delta \vec{r}_{D} ; \quad \vec{\rho}_{2}=\vec{\rho}_{1}+\Delta \vec{\rho}_{12} ; \quad \vec{\rho}_{R 2}=\vec{\rho}_{1}+\Delta \vec{\rho}_{R}
\end{gathered}
$$

With this in mind, we determine the path differences $\Delta_{1}$ and $\Delta_{2}$ : 


$$
\begin{aligned}
& \Delta_{1}=\vec{\rho}_{0} \cdot \vec{r}_{0}+\vec{\rho}_{1} \cdot \vec{r}_{1}-\vec{r}_{3} \cdot \vec{\rho}_{0}-\vec{r}_{4} \cdot \vec{\rho}_{R 1} \\
& \Delta_{2}=\vec{r}_{5} \cdot \vec{\rho}_{0}+\vec{r}_{6} \cdot \vec{\rho}_{2}-\vec{r}_{7} \cdot \vec{\rho}_{0}-\vec{r}_{8} \cdot \vec{\rho}_{R 2}=\left(\vec{r}_{0}+\vec{W}_{B}\right) \cdot \vec{\rho}_{0}+ \\
& +\left(\vec{r}_{1}-\Delta \vec{r}_{B}\right) \cdot\left(\vec{\rho}_{1}+\Delta \vec{\rho}_{12}\right)-\vec{\rho}_{0} \cdot\left(\vec{r}_{3}+\vec{W}_{D}\right)-\left(\vec{\rho}_{R 1}+\Delta \vec{\rho}_{R}\right) \cdot\left(\vec{r}_{4}-\Delta \vec{r}_{D}\right)
\end{aligned}
$$

Substituting these expressions into equation (16), we obtain:

$$
\begin{aligned}
& \vec{r}_{0} \cdot \vec{\rho}_{0}+\vec{\rho}_{1} \cdot \vec{r}_{1}-\vec{r}_{3} \cdot \vec{\rho}_{0}-\vec{r}_{4} \cdot \vec{\rho}_{R 1}-\vec{r}_{0} \cdot \vec{\rho}_{0}+\vec{W}_{B} \cdot \vec{\rho}_{0}-\vec{r}_{1} \cdot \vec{\rho}_{1}+\Delta \vec{r}_{B} \cdot \vec{\rho}_{1}- \\
& -\Delta \vec{\rho}_{12} \cdot \vec{r}_{6}+\vec{\rho}_{0} \cdot \vec{r}_{3}-\vec{W}_{D} \cdot \vec{\rho}_{0}+\vec{\rho}_{R 1} \cdot \vec{r}_{4}-\vec{\rho}_{R 1} \cdot \vec{r}_{4}-\vec{\rho}_{R 1} \cdot \Delta \vec{r}_{D}+\vec{\rho}_{R} \cdot \vec{r}_{8}= \\
& =\left(\vec{W}_{B} \cdot \vec{\rho}_{0}+\Delta \vec{r}_{B} \cdot \vec{\rho}_{1}\right)-\left(\vec{W}_{D} \cdot \vec{\rho}_{0}+\Delta \vec{r}_{D} \cdot \vec{\rho}_{R 1}\right)-\left(\Delta \vec{\rho}_{12} \cdot \vec{r}_{6}-\Delta \vec{\rho}_{R} \cdot \vec{r}_{8}\right)=N \lambda
\end{aligned}
$$

Directions of vectors $\Delta \vec{\rho}_{12}, \vec{r}_{6}$ and $\Delta \vec{\rho}_{R}, \vec{r}_{8}$ are orthogonal in practice, therefore their products are equal to zero. Vectors $\vec{W}_{B}, \vec{W}_{D}$ and $\vec{\rho}_{0}$ are parallel, therefore their products $\vec{W}_{B} \cdot \vec{\rho}_{0}=W_{B}$ and $\vec{W}_{D} \cdot \vec{\rho}_{0}=W_{D}$. The path difference in the object beams during the time between the first and second exposure is described by the terms in the first brackets. The similar path difference in the reference beams is described by the second bracket.

For information recorded in reflected light, the reference beam does not change. The interference fringes observed in reflected light are described by the equation:

$$
\left(W_{B}+\Delta \vec{r}_{B} \cdot \vec{\rho}_{1}\right)=U_{B} \cos \alpha+V_{B} \cos \beta+W_{B}(1+\cos \gamma)=N \lambda
$$

The pattern recorded in the light passing through the hologram is described by the equation:

$$
\begin{aligned}
& \left(W_{B}+\Delta \vec{r}_{B} \cdot \vec{\rho}_{1}\right)-\left(W_{D}+\Delta \vec{r}_{D} \cdot \vec{\rho}_{R}\right)= \\
& {\left[U_{B} \cos \alpha+V_{B} \cos \beta+W_{B}(1+\cos \gamma)\right]-} \\
& -\left[U_{D} \cos \alpha_{R}+V_{D} \cos \beta_{R}+W_{D}\left(1+\cos \gamma_{R}\right)=N \lambda\right.
\end{aligned}
$$

In the course of the experiment, the angles $\alpha, \beta, \gamma$ are set (when choosing the direction of observation). The indicatrix of the scattering of the surface determines the angles $\alpha_{R}, \beta_{R}$, and $\gamma_{R}$. When processing interference patterns, the order of the $\mathrm{N}$ band is determined. Using equations (20) and (21), the components of the displacement vector $\mathrm{U}, \mathrm{V}$ and $\mathrm{W}$ are calculated. When studying flat surfaces $\alpha_{R}=90^{\circ}, \beta_{R}=90^{\circ}$, and $\gamma_{R}=0$, the equation (21) takes the form:

$$
U_{B} \cos \alpha+V_{B} \cos \beta+W_{B}(1-\cos \gamma)-2 W_{D}=N \lambda
$$

To determine the components of the displacement vector in reflected light, two patterns are recorded. The directions of their observation are symmetrical relative to the normal to the hologram. If the directions of observation are in the plane ${ }^{X O Z}$, then the resulting equations have the form:

$$
U_{B} \sin \gamma+W_{B}(1+\cos \gamma)=N_{1} \lambda
$$




$$
-U_{B} \sin \gamma+W_{B}(1+\cos \gamma)=N_{2} \lambda,
$$

Here $\gamma$ is the angle between the direction of observation and the normal to the surface of the product, $N_{1}$ and $N_{2}$ are the orders of the bands in the patterns observed at the angles $\gamma$ and ${ }^{-\gamma}$. The displacement component lying in the plane of the sample and the recording plane shall be determined by the equation:

$$
U_{B}=\frac{\left(N_{1}-N_{2}\right) \lambda}{2 \sin \gamma}
$$

The determination of the displacement vector components lying in the plane along the interference patterns of bands recorded in the light passing through the hologram, is possible only with a small gap between the investigated surface and the recording medium.

\section{Results}

The method of holographic interferometry in colliding beams was used to determine the permissible distance between dowels. The reflectivity of the wood surface is small, so a reflective layer of aluminum powder was applied to the surface of the wooden beam. A dowel was hammered into the wooden beam. High-resolution holographic photographic plate was fixed on the surface of the beam. The photographic plate was exposed at two different loads applied to the dowel along the wood fibers. After the processing of the photographic plate, the patterns of interference fringes from two directions symmetrical with respect to the normal to the plate were recorded. Photos of these patterns are shown in Figure 2. The observation plane coincides with the direction of the wood fibers and is normal to the sample surface.

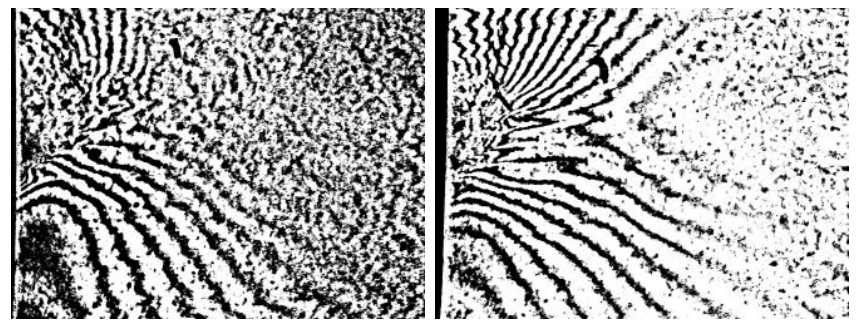

Fig. 2. Photographs of patterns of bands.

Photographs of the interferograms shown in Figure 2 were recorded when studying the strain state of wood near the dowel with the largest transverse dimension of $16 \mathrm{~mm}$. When

processing optical information, diagrams $N^{*}=\frac{N_{1}-N_{2}}{2}$ were plotted along sections parallel to the fibers of wood. The value $N^{*}$ is proportional to the component of the displacement vector $U$. The distance between the sections is $6 \mathrm{~mm}$. To build the diagrams, we used the expression (24). Having made a linear assumption, it is possible to calculate a value proportional to the component of the shear deformation, 


$$
\frac{d U}{d y} \propto \frac{\Delta N}{d}
$$

Here, $d$ is the distance between the sections; $\Delta N$ - the difference between the values $N^{*}{ }_{1}-N^{*}{ }_{2}$

2 calculated in different sections at points with the same coordinate $x$. The axis $x$ coincides with the direction of the wood fibers. It is horizontal in Figure 2. It follows from (25) that $\ln \frac{d U}{d y} \propto \ln \Delta N-\ln d$

Figure 3 shows the diagram $\ln \Delta N$, as you can see, it is almost straight.

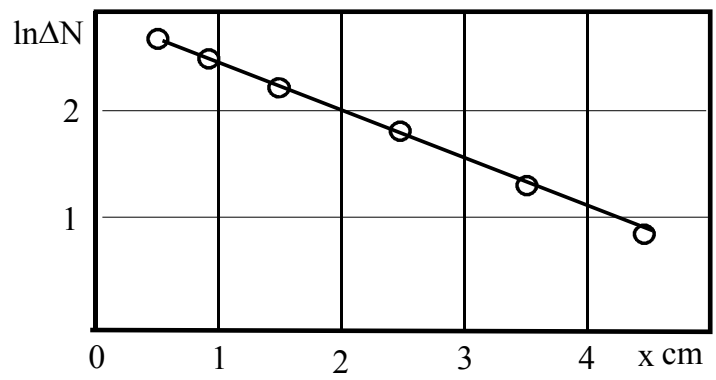

Fig. 3. Diagram $\ln \Delta N$

So, $\frac{d U}{d y}$ and $\Delta N$ decrease exponentially [12] with distance from the axis of the dowel. Therefore, it can be assumed that the shear deformation component varies according to the law: $\frac{d U}{d y}=A e^{-\frac{x}{\tau}}$, here $\tau_{\text {- distance at which }} \ln \frac{d N}{d y}$ and $\ln \Delta N$ decreases by unit (attenuation constant). For a dowel with a width of $16 \mathrm{~mm}, \tau=-1.95 \mathrm{~cm}$. The attenuation constant for a $14 \mathrm{~mm}$ dowel was determined in the same way, it turned out to be $1.75 \mathrm{~cm}$.

\section{Conclusion}

For the reliable work of dowels, it is necessary that in the region of intersection of disturbances from adjacent dowels, the magnitude of the shear stresses does not exceed $2 \%$ of the maximum value. This is achieved at a distance equal $4 \tau$, therefore, the distance between the axes of adjacent dowels should be equal $8 \tau$. For a dowel in width of $16 \mathrm{~mm}$, the distance between the axes should be $16 \mathrm{~cm}$, and for $14 \mathrm{~mm}$ dowel $-14 \mathrm{~cm}$. The developed technique allows determining the permissible distances between connecting elements in wooden structures. 


\section{References}

1. V.I. Zhadanov, P.A. Dmitriev, V.N. Shvedov, G.A. Stolpovskiy, D.A. Ukrainchenko, RU Patent 2353830 (2007). (in Russian)

2. A.M. Popov, V.B. Zinovyev, Vestnik of TSUAB 2, 235-238 (2012). (in Russian)

3. V.I. Zhadanov, M.A. Arkaev, V.G. Kotlov, Industrial and Civil Engineering 11, 5-11 (2017). (in Russian)

4. Q.K. Cao, H.M. Xie, Experimental Mechanics, 1-16 (2018).

5. Y.L. Dong, B. Pan, Experimental Mechanics 8, 1161-1161 (2017).

6. T.E.J. Edwards, F. Di Giacchino, H.P. Springbett, R.A. Oliver, Experimental Mechanics 9, 1469-1482(2017).

7. Yu.N. Denisyuk, DAN SSSR 144 (1962). (in Russian)

8. Yu.N. Denisyuk, Principy golografii [Principles of holography] (Leningrad, 1979). (in Russian)

9. G.V. Ostrovskaya, Journal of Technical Physics 6, 1-16 (2016). (in Russian)

10. A.M. Popov, V.B. Zinovyev, L.A. Spodareva, Scientific problems of transport in Siberia and the Far East 3, 77-80 (2015). (in Russian)

11. S.I. Gerasimov, V.A. Zhilkin, Applied Mechanics and Technical Physics 3, 176-182 (2006). (in Russian)

12. A.G. Demeshkin, V.M. Kornev, Mechanics of Composite Materials 2, 319-324 (1981). (in Russian) 